\title{
Performance-based risk-sharing arrangements for devices and diagnostics in the United States: a systematic review
}

Yilin Chen, MPH, and Josh J Carlson, PhD, MPH

\section{What is already known about this subject}

- Performance-based risk-sharing arrangements (PBRSAs) have become an option for medical technology contracting in many health care systems.

- In the US private sector, considerable interest remains in outcomes-based agreements; however, most of the published analyses have focused on pharmaceuticals.

\section{ABSTRACT \\ BACKGROUND: Performance-based risk- sharing arrangements (PBRSAs) have continued to emerge and evolve over the last 2 decades. To date, most of the attention and available literature have focused on pharmaceuticals.}

OBJECTIVE: To assess the current status and trends regarding the use of PBRSAs for diagnostics and devices in the United States.

METHODS: We reviewed publicly available PBRSAs for diagnostics and devices using the University of Washington Performance Based Risk Sharing Database. We augmented the review using PubMed, Google, and

\section{What this study adds}

- The key payer groups involved with PBRSAs applied to devices were hospitals, integrated health delivery systems, and public and private payers; in the diagnostics space, private health plans and public payers, such as the Centers for Medicare \& Medicaid Services, form the payer mix.

- Diagnostics indirectly affect health outcomes by informing clinical decision making; therefore, performance-based arrangements for diagnostics may be most applicable for short-term process metrics rather than longer-term clinical outcomes.

- This study found substantial activity overall with increasing use of PBRSAs since 2014, especially for performancelinked reimbursement and coverage with evidence development, only with research arrangements.

payer and industry websites. Key words and phrases such as outcomes-based, valuebased, coverage with evidence development, performance-based, and risk-sharing were used in combination with device or diagnostic. To characterize arrangements in terms of product and market attributes, we extracted data for each product, including arrangement descriptions, arrangement type, year, therapeutic area, product manufacturer, payer, and product type. Arrangements were analyzed using descriptive statistics.

RESULTS: Fifty-two arrangements were identified between the years 2001 and 2019, with 30 (57.7\%) for devices and 22 (42.3\%) for diagnostic tests. Among these, 23 (44.2\%)

\section{Author affiliations \\ Yilin Chen, MPH, and Josh J Carlson, PhD, $\mathrm{MPH}, \mathrm{CHOICE}$ Institute, School of Pharmacy, University of Washington, Seattle.}

AUTHOR CORRESPONDENCE:

Josh J Carlson, 206.543.9649;

carlsojj@uw.edu were coverage with evidence development (CED), only in research; 17 (32.7\%) were performance-linked reimbursement $(P L R)$; and 12 (23.1\%) were CED, only with research. The majority of arrangements for devices were developed in cardiology $(12,40 \%)$, endocrinology $(4,13.3 \%)$, and radiology ( 3 , $10 \%)$. Most of arrangements for identified diagnostic tests were in oncology $(17,77.3 \%)$. Over time, there has been a trend towards increasing adoption of PLR and CED, only with research, especially since 2014 .

CONCLUSIONS: This is the first study to comprehensively review PBRSA arrangements for diagnostics and devices in the United States. Our findings demonstrated that there is 
substantial PBRSA activity for devices and diagnostics, and the pace of PBRSA adoption appears to be increasing in terms of frequency and variety. These arrangements have implications for managed care into the future as the health care system shifts towards value-based care and value-based pricing to contain cost for payers and ensure value in the patient populations.

Performance-based risk-sharing arrangements (PBRSAs) have grown at a variable pace across countries over the past 20 years. PBRSAs (also known as patient access schemes and managed entry arrangements) can be defined as arrangements between a payer and a pharmaceutical, or device manufacturer, where the level or continuation of reimbursement is linked to the real-world performance of medical products (ie, health or economic outcomes), in a defined patient population over a specified period of time. ${ }^{1}$ These arrangements have gained enthusiasm in the United States in response to a number of factors: (1) uncertainty in the real-world performance (effectiveness and safety) of the medical product in a given patient population at market introduction; (2) cost pressures on manufacturers and payers with increasing research and development costs; (3) the emergence of new technologies, including pharmaceuticals, diagnostics, and devices at a faster pace; and (4) the general expansion of the use of real-world data for approvals by the US Food and Drug Administration., ${ }^{2,3}$

In this environment, PBRSAs that link coverage and reimbursement to real-world outcomes can alter or shift the distribution of risks among parties, allowing an additional avenue for negotiation and balance between interacting stakeholders. Well-designed PBRSAs can offer multiple benefits for payers, such as cost containment, real-world evidence generation, and reductions in uncertainty of longterm performance, as well as for manufacturers in terms of earlier and expanded market access. ${ }^{4,5}$

Compared with PBRSAs for pharmaceuticals, arrangements for devices and diagnostics may be unique for a few key reasons. There are typically a different mix of payers and payer dynamics for medical devices or diagnostic tests, with more involvement by hospitals and integrated health care delivery systems. The nature of product success or failure is different with potential short-term processrelated outcomes offering an early performance metric suitable for use in PBRSAs. Devices and diagnostics typically have lower budget impacts than new drugs, as well as different intellectual property and patent environments. Finally, as we discussed briefly in previous work on PBRSAs, devices and diagnostics typically have a less well-developed evidence base at the time of market entry. These various product and market dynamics for devices and diagnostics may lead to different incentives, risk tolerances, and, ultimately, different trends in terms of arrangement types and adoption rates than those for pharmaceuticals.

Very little has been published to date regarding PBRSAs for devices and diagnostics, especially in the United States. As these arrangements continue to gain momentum for use, a review of the subject is warranted in order to inform relevant stakeholders (manufacturers, payers, and policymakers) about the current status and key trends. Our objective was to review the initiation of PBRSAs for devices and diagnostics during the past 2 decades in the United States.

\section{Methods}

We reviewed publicly available PBRSAs for diagnostics and devices, using the University of Washington Performance Based Risk Sharing (PBRS) Database. We augmented the review using PubMed, Google, and payer and industry websites, including the top 15 medical device and diagnostics companies by revenue and the top 10 health insurance companies by market value. The review covered arrangements initiated over the past 2 decades (January 2001-September 2020). The last search date was September 30, 2020. Key words and phrases such as outcomes-based, value-based, coverage with evidence development, performance-based, and risk-sharing were used in combination with device or diagnostic.

To characterize arrangements in terms of product and market attributes, we extracted the following data for included arrangements: year of initiation, type of medical product (ie, diagnostics or devices), manufacturer and payer, therapeutic area, and coverage and reimbursement consequences. We further characterized arrangements according to our previously published taxonomy. ${ }^{4,6}$ Three main categories were used: (1) coverage with evidence development (CED), only in research; (2) CED, only with research; and (3) performance-linked reimbursement (PLR). CED is defined as coverage that is conditioned on the collection of additional evidence from predefined study to support continuation, expansion, or withdrawal of coverage. For the "only in research" variety, coverage is conditioned on individual participation in research (ie, only participated patients are covered). For the "only with research" variety, coverage is conditioned on the conduct of a study in the payer patient population, but individual patients do not need to participate to be covered (ie, a sample of patients are involved in a study, but all target patient population are covered). Finally, PLR occurs when the reimbursement 


\section{FIGURE 1 Number of New Arrangements by Arrangement Categories (2001-2019)}

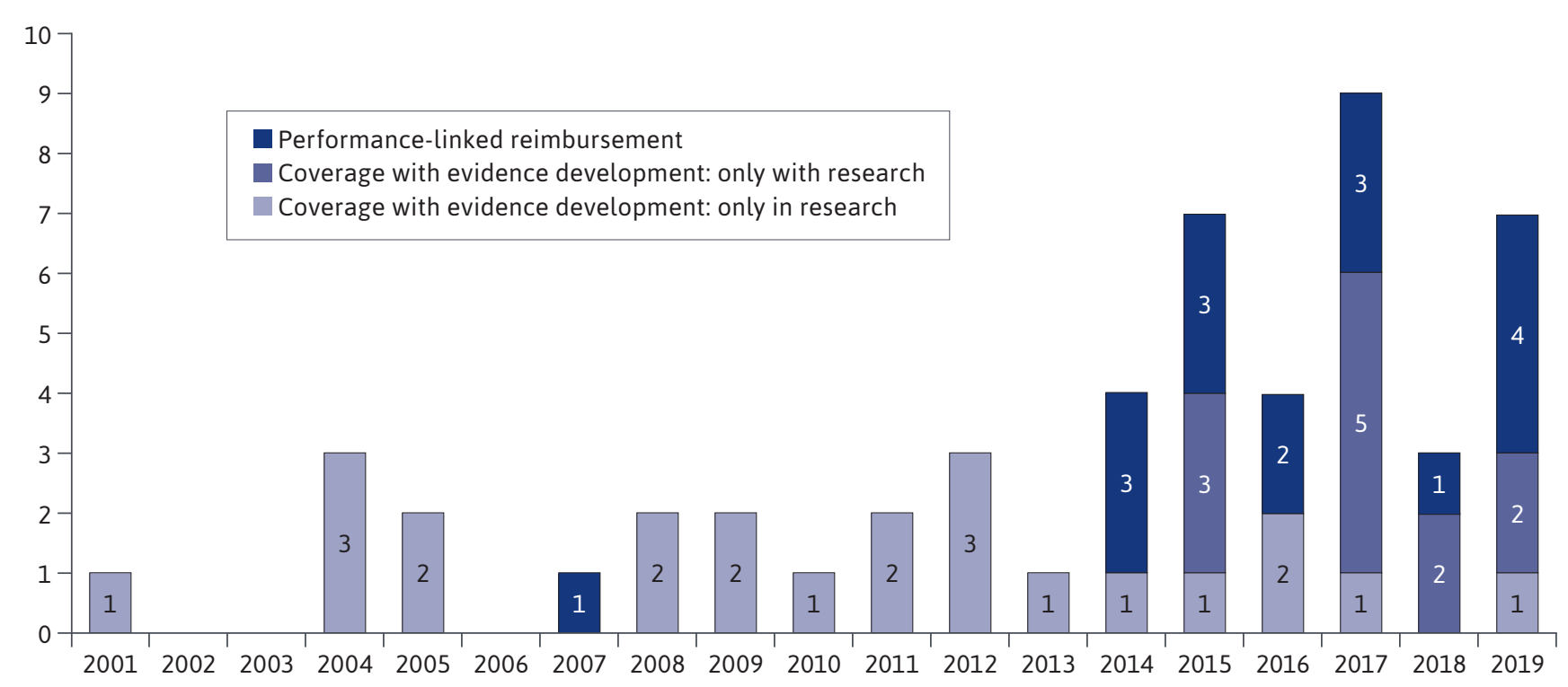

level for a covered product is linked to a measure of clinical outcomes. ${ }^{4,6}$

The analytic approach used descriptive statistics, including frequencies and proportions. Macro-level trends were identified that related to the overall PBRSA adoption over time, the type of arrangements, product types, and therapeutic areas. All analyses were performed in Microsoft Excel 2017.

\section{Results}

This review identified 52 performance-based arrangements that were initiated between 2001 and 2019. Among these, 23 (44.2\%) were CED, only in research; 17 (32.7\%) were PLR; and 12 (23.1\%) were CED, only with research. Figure 1 shows that, before 2014, the majority of arrangements were CED, only in research. Over time, there has been a trend towards increasing adoption of arrangements with PLR and CED, only with research components, especially since 2014. In addition, more than half of the total arrangements found $(30,57.7 \%)$ were initiated in the past 5 years (between 2015 and 2019).

The distribution of arrangements by product type over time is provided in Supplementary Figure 1 (available in online article). More than half of arrangements were for devices $(30,57.7 \%)$, with the remaining established for diagnostic tests $(22,42.3 \%)$. The majority of arrangements for devices were developed in cardiology $(12,40 \%)$, followed by endocrinology $(4,13.3 \%)$ and radiology $(3,10 \%)$. Unlike devices, oncology was the top therapeutic area of interest for diagnostics, with 17 cases (77.3\%), followed by neurology (2, 9.1\%; Figure 2).

Most of the arrangements were part of the CED arrangements at the Centers for Medicare \& Medicaid Services (CMS) or CMS contractors (Supplementary Figure 2 and Supplementary Tables 1 and 2 , available in online article). Major payers were characterized by private or commercial insurance companies (ie, Aetna, UnitedHealthcare, Blue Cross and Blue Shield [BCBS], and Capital District Physicians' Health Plan [CDPHP]); integrated health care delivery systems (ie, Atrium Health and Fairview Health Services); and various hospitals. As of September 30, 2020, of 52 arrangements, 34 (65.4\%) were CEDs published on the CMS website and included 20 (58.8\%) covered through national coverage determinations (NCDs) at CMS and 14 (41.2\%) local coverage determination (LCDs) by the Medicare contractor Palmetto GBA. ${ }^{7}$ The remaining arrangements $(18,34.6 \%)$ were identified from websites of private payers.

Among the CEDs issued by public payers, the main therapeutic areas were oncology $(16,47.1 \%)$ and cardiology (9, 26.5\%). Most arrangements in oncology were cancer classifier assays, genomic profiling or score, or epigenetic testing. These classifications are consistent with the emerging trend of using genomic profiling and genetic testing 


\section{FIGURE 2 Arrangements for Devices and Diagnostics by Therapeutic Areas ( $\mathrm{N}=52)$}

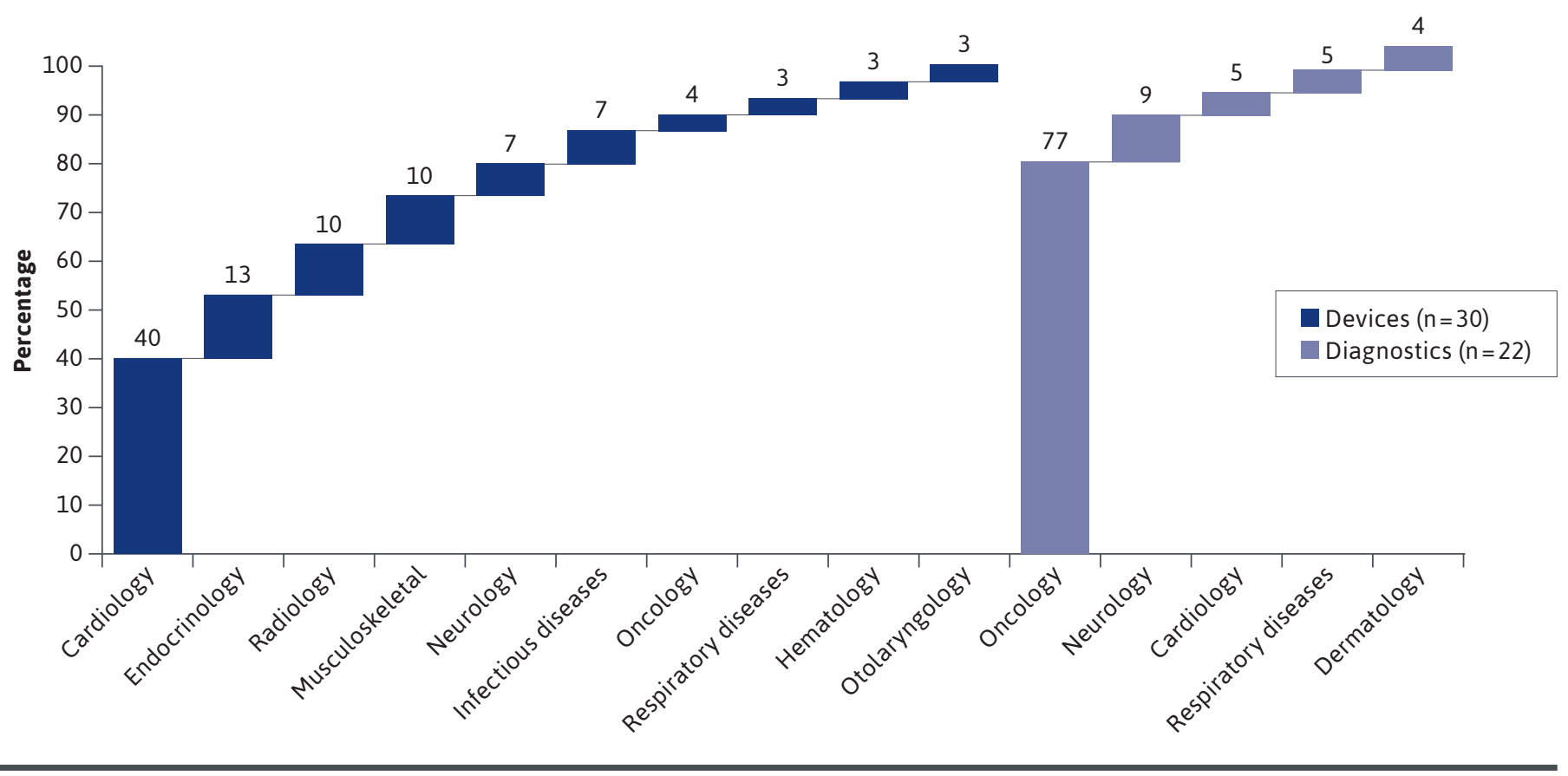

for precision medicine diagnostics. For instance, the first classifier assays with CED arrangements were released in 2015 for ConfirmMDx Epigenetic Molecular Assay, Prolaris, Decipher, and Oncotype DX Prostate Cancer Assay for prostate cancer. More products were added recently for different cancers. In addition, most arrangements issued by private payers were device related, except 1 diagnostic test (ie, Oncotype DX for breast cancer).

Of all arrangements, 5 (9.6\%) were issued by private or commercial insurance companies, with 4 insulin pump devices for diabetes manufactured by Medtronic and another connected with UnitedHealthcare's arrangement to tie reimbursement of Genomic Health's Oncotype DX assay to a program of data collection in women considering adjuvant breast cancer therapy. Finally, arrangements in use by hospitals $(11,21.2 \%)$ and integrated health systems $(2,3.8 \%)$ covered a wide range of disease areas for medical devices.

We identified multiple PBRSAs for the same product under the same disease area but with different payers. For example, Minimed 670G System by Medtronic, an insulin pump system for diabetes, was identified in PLR arrangements with Aetna, BCBS of Minnesota, and CDPHP. The PLR arrangement required the reimbursement to be tied to successfully meeting agreed upon clinical improvement thresholds for enrolled members. Moreover, multiple PBRSAs for the same product but for different disease areas were identified. For instance, fluorodeoxyglucose positron emission tomography was identified as a CED, only in research, on the CMS website for cognitive impairment and other identified neurodegenerative diseases. It was also covered through CED for brain, cervical, ovarian, pancreatic, small cell lung, and testicular cancers.

\section{Discussion}

Our study was the first to comprehensively review existing PBRSAs for devices and diagnostics in the United States. Overall, the adoption of PBRSAs appears to have grown in recent years, with an increasing number and types of arrangements established since 2014. The distribution of arrangements is slightly skewed toward devices vs diagnostics. The most common therapeutic area for devices was cardiology, whereas the majority of arrangements for diagnostics was related to oncology.

A key finding from our study is the increasing use of PLR and CED, only with research arrangements, in addition to $\mathrm{CED}$, only in research. The increase in the use of CED, only 
with research, arrangements was mainly driven by CMS, which published the most arrangements among all payers. CMS generally issues 2 types of Medicare coverage determination processes (NCDs and LCDs), with the purpose of generating more evidence to inform coverage decisions.

In the absence of a national coverage policy, a technology may be covered at the discretion of the Medicare contractors through an LCD. ${ }^{7}$ PLR arrangements, in contrast to CED arrangements, were implemented with private payers and hospitals. This trend is consistent with increased interest and adoption of PBRSAs by private US insurers for pharmaceuticals, as well as continued movement towards value-based care and value-based pricing to contain costs for payers and ensure value in the payer populations. ${ }^{5,8}$

Our analysis showed that private payers, including health plans and commercial insurance companies, developed PBRSAs mainly for medical devices rather than diagnostics. An exception was the Oncotype DX Breast Recurrence Score, which quantifies the risk of distant recurrence and the likelihood of chemotherapy benefit. PLRs used for devices can provide benefits in a few key ways: (1) plan members have improved access to advanced devices, (2) risks from uncertainty in clinical outcomes are shared between payers and manufacturers, and (3) manufacturers can secure early and expanded market access for their new devices.

Similarly, hospitals and integrated health systems have set up outcomes guarantee arrangements-a form of PLRwhich typically include device manufacturers that provide refunds or rebates if a device does not perform as agreed to in terms of clinical or economic outcomes within certain time frames. With the expanded implementation of electronic medical record systems and the enhanced ability to analyze big data, it may become easier to access and track real-world outcomes and use of care in hospitals and integrated delivery systems.

As with devices, previous literature regarding the application of PBRSAs to diagnostics is scarce, since much of the focus has been on pharmaceuticals because of their larger share of health care spending and the increasing focus on rising drug costs. ${ }^{9}$ Diagnostics occupy a unique position in clinical care because they indirectly affect health outcomes by informing clinical decision making rather than directly affecting health outcomes. Therefore, PBRSAs for diagnostics may be most applicable for short-term process metrics rather than longer-term clinical outcomes.

Our analysis showed that a key player in the diagnostics arena has been Palmetto GBA, a CMS contractor, with its data development program, which is a version of CED. This program has implemented a few PBRSAs for genomic classifiers and genetic tests. These arrangements vary by product type but typically involve annual or biannual review of required manufacturer data submissions from prospective clinical studies on predefined patient outcomes by the contractor for continued coverage..$^{10}$

Some programs include good performance rewards for manufacturers in the form of expanded sets of approved providers who can use the diagnostic or expanded patient cohorts eligible for product use, which allows for increased market penetration. Prolaris Prostate Cancer Genomic Assay, for instance, gained an initial LCD among patients with needle biopsy proven early-stage prostate cancer in 2015. Two years later, Palmetto GBA expanded its coverage to prostate cancer patients with favorable intermediate risk disease (Supplementary Table 1). ${ }^{11,12}$ Palmetto GBA was cited in 14 arrangements to date through our search. The apparent success of this program suggests that this is potentially a reproducible and efficient approach, incentivizing evidence generation in the diagnostics space.

A previous review of PBRSAs noted that NCDs have experienced some difficulty in conducting associated research because of the lack of dedicated funding streams. ${ }^{1,13} \mathrm{~A}$ similar challenge was identified in a review of CEDs for medical devices in France. Since 2007, the French National Committee of Medical Devices and Health Technologies has proposed the creation of CEDs for innovative devices. However, only 2 devices have benefited 5 years after the introduction of CED arrangements because of the lack of sustainable funding for future studies. The committee proposed a possible solution that involved hospital-based research programs funded by the Ministry of Health. ${ }^{14}$

Another common challenge in developing PBRSAs is to identify measurable outcomes and to track success. ${ }^{5} \mathrm{~A}$ recent activity launched by the Pushing the Boundaries of Cost and Outcome Analysis of Medical Technologies project has published comprehensive materials on how to conduct health technology assessments for medical devices, such as use of real-world evidence and surrogate outcomes. ${ }^{15}$ The primary aim of this project is to explore CED arrangements currently applied to medical devices in Europe. Seventytwo arrangements from 8 countries have been in operation between 2015 and 2020. This work aligns with our study and supports the conclusion that there is substantial activity and use of PBRSAs for devices and diagnostics in Europe, as well as in the United States.

\section{LIMITATIONS}

This study has some limitations to consider. First, we were only able to collect information on publicly available cases. Except for the CMS website, there was no centralized 
platform for publishing these arrangements. Our PBRS Database at the University of Washington has regularly updated cases from different sources, which enabled us to fill this knowledge gap. However, since some arrangements are confidential, our analysis likely underestimated their true prevalence and impact. ${ }^{16}$ It may lead to a biased sample if the adoption pace or type of arrangement differs for confidential vs publicly available arrangements. Moreover, there could be incomplete data for arrangements made in 2019-2020 because of the delay in time between initial contracting and final publication.

In addition, since most arrangements did not report term or result, such data were not captured here. Our findings may not reflect the actual level of interest in PBRSAs, which may vary between manufacturers and payers, if they are not directly represented by published articles or news.

\section{Conclusions}

Despite challenges, the adoption of PBRSAs for devices and diagnostics have continued to gain interest among in different payers in the United States. PLR; CED, only with research arrangements; and CED, only in research, are the major types of arrangements for devices and diagnostics, with the level of activity differing between public and private payers and hospitals. Continued effort is required from various stakeholders to improve the design, process, efficiency, and effectiveness of PBRSA arrangements.

\section{DISCLOSURES}

No funding supported this study. The authors have nothing to disclose.

\section{REFERENCES}

1. Garrison LP Jr, Towse A, Briggs A, et al. Performance-based risk-sharing arrangements-good practices for design, implementation, and evaluation: report of the ISPOR Good Practices For Performance-Based Risk-Sharing Arrangements Task Force. Value Health. 2013;16(5):703-19.

2. DiMasi JA, Hansen RW, Grabowski HG. The price of innovation: new estimates of drug development costs. J Health Econ. 2003;22(2):151-85.

\section{Baker LC, Atlas SW, Afendulis CC.}

Expanded use of imaging technology and the challenge of measuring value. Health Aff (Millwood). 2008;27(6):1467-78.

4. Carlson JJ, Gries KS, Yeung K, Sullivan SD, Garrison LP Jr. Current status and trends in performance-based risksharing arrangements between healthcare payers and medical product manufacturers. Appl Health Econ Health Policy. 2014;12(3):231-38.

5. Dubois RW, Westrich K, Buelt L. Are value-based arrangements the answer we've been waiting for? Value Health. 2020;23(4):418-20.

6. Carlson JJ, Sullivan SD, Garrison LP Jr, Neumann PJ, Veenstra DL. Linking payment to health outcomes: a taxonomy and examination of performance-based reimbursement schemes between healthcare payers and manufacturers. Health Policy. 2010;96(3):179-90.

7. Centers for Medicare \& Medicaid Services. How to request an NCD. 2020. Accessed October 17, 2020. https://www. cms.gov/Medicare/Coverage/Deter minationProcess/howtorequestanNCD

8. Maddox KJ, Bleser WK, Crook HL, et al. Advancing value-based models for heart failure: a call to action from the Value in Healthcare Initiative's Value-Based Models Learning Collaborative. Circ Cardiovasc Qual Outcomes. 2020;13(5):e006483.

9. IQVIA Institute for Human Data Science. Medicine use and spending in the U.S.: a review of 2018 and outlook to 2023. May 9, 2019. Accessed October 18, 2020. https://www.iqvia.com/ insights/the-iqvia-institute/reports/ medicine-use-and-spending-in-the-us-areview-of-2018-and-outlook-to-2023
10. Centers for Medicare \& Medicaid Services. Guidance for the public, industry, and CMS staff: coverage with evidence development. November 20, 2014. Accessed October 17, 2020. https://www.cms.gov/medicare-coverage-database/details/ medicare-coverage-document-details. aspx?MCDId=27

11. Centers for Medicare \& Medicaid Services. Medicare Coverage Database. Local Coverage Determination (LCD): MolDX: Prolaris ${ }^{\mathrm{TM}}$ Prostate Cancer Genomic Assay for Men with Favorable Intermediate Risk Disease (L37043). 2017. Accessed October 17, 2020. https://www. cms.gov/medicare-coverage-database/ reports/local-coverage-proposed-lcdscontractor-report.aspx?contractorName $=2 \&$ contractorNumber=all\&proposedSta tus=all

12. Centers for Medicare \& Medicaid Services. Medicare Coverage Database. Local Coverage Determination (LCD): MolDX: Prolaris ${ }^{\mathrm{TM}}$ Prostate Cancer Genomic Assay (L35869). 2015. Accessed October 17, 2020. https://www.cms.gov/ medicare-coverage-database/view/lcd. aspx?LCDId=35869

13. Carlson JJ, Chen S, Garrison LP Jr. Performance-based risk-sharing arrangements: an updated international review. Pharmacoeconomics. 2017;35(10):1063-72.

14. Martelli N, van den Brink H, Borget I. New French coverage with evidence development for innovative medical devices: improvements and unresolved issues. Value Health. 2016;19(1):17-19.

15. The COMED Project. WP7 coverage with evidence development for medical devices. 2020. Accessed October 17, 2020. https://www.comedh2020.eu/wps/wcm/ connect/site/comed/home/project/ work+packages/wp7.

16. Mahendraratnam N, Sorenson C, Richardson E, et al. Value-based arrangements may be more prevalent than assumed. Am J Manag Care. 2019;25(2):70-76. 